\title{
Immunogenicity of Pfizer mRNA COVID-19 Vaccination Followed by J\&J Adenovirus COVID-19 Vaccination in Two Patients with Chronic Lymphocytic Leukemia
}

\author{
Zoe L. Lyski $\left(\mathbb{D},{ }^{1}\right.$ Myung Sun Kim ${ }^{1}{ }^{2}$ David Xthona Lee ${ }^{1},{ }^{1}$ David Sampson, ${ }^{2}$ \\ Hans-Peter Raué, ${ }^{3}$ Vikram Raghunathan, ${ }^{2}$ Debbie Ryan, ${ }^{2}$ Amanda E. Brunton (D), \\ Mark K. Slifka, ${ }^{3}$ William B. Messer, ${ }^{1,3,5}$ and Stephen E. Spurgeon ${ }^{2}{ }^{2}$ \\ ${ }^{1}$ Department of Molecular Microbiology \& Immunology, Oregon Health \& Science University (OHSU), Portland, OR 97239, USA \\ ${ }^{2}$ Knight Cancer Institute, Oregon Health \& Science University (OHSU), Portland, OR 97239, USA \\ ${ }^{3}$ Division of Neuroscience, Oregon National Primate Research Center, Oregon Health \& Science University, Beaverton, \\ OR 97239, USA \\ ${ }^{4}$ OHSU-PSU School of Public Health, Portland, OR 97239, USA \\ ${ }^{5}$ Department of Medicine, Division of Infectious Diseases, Oregon Health \& Science University (OHSU), \\ Portland, OR 97239, USA
}

Correspondence should be addressed to Stephen E. Spurgeon; spurgeos@ohsu.edu

Received 9 November 2021; Accepted 14 January 2022; Published 4 February 2022

Academic Editor: Pier Paolo Piccaluga

Copyright (C) 2022 Zoe L. Lyski et al. This is an open access article distributed under the Creative Commons Attribution License, which permits unrestricted use, distribution, and reproduction in any medium, provided the original work is properly cited.

Individuals with chronic lymphocytic leukemia (CLL) have significant immune disfunction, often further disrupted by treatment. While currently available COVID-19 vaccinations are highly effective in immunocompetent individuals, they are often poorly immunogenic in CLL patients. It is important to understand the role a heterologous boost would have in patients who did not respond to the initial two-dose mRNA vaccine series. SARS-CoV-2 specific immune responses, including antibodies and memory B-cells, CD4 and CD8 T-cells were assessed prior to vaccination, as well as postinitial vaccination series and post-third dose in two subjects. One subject seroconverted, had RBD-specific memory B-cells and spike-specific CD4 T-cells while the other did not. Both subjects had a spike-specific CD8 T-cell response after the original mRNA vaccination series that was further boosted after the third dose or remained stable. The results of this study, however small, are especially promising to CLL individuals who did not seroconvert following the initial mRNA vaccination series.

\section{Introduction}

Chronic lymphocytic leukemia (CLL) is characterized by the monoclonal proliferation of dysfunctional B-cells, leading to a broad range of immune defects. CLL patients face significant risk of morbidity and mortality from infections [1], including from SARS-CoV-2, the causative agent of COVID-19 [2]. Vaccines can be instrumental in mitigating the risk of infections in CLL; however, responses to vaccination are highly variable and significantly influenced by CLL disease status, baseline characteristics, types of vaccine, and active CLL therapy [3].
Although current COVID-19 vaccines elicit robust immunity in immunocompetent hosts [4], the antibody response in CLL patients is highly variable [5-7] and particularly poor in patients with low total immunoglobulin levels, those that have had anti-CD20 monoclonal antibodies within the past year, or those that are undergoing active therapy with agents such as Bruton's tyrosine kinase inhibitors (BTKi). The best responses to date have been in CLL patients who are in remission and/or years out from active treatment.

Given decreased vaccine efficacy in CLL, an additional dose of vaccine may be beneficial in CLL patients, especially 
given the rise of variants of concern (VoCs). Initial data from solid organ transplant recipients on immunosuppression as well as individuals with solid tumors on active therapy showed a role for additional vaccination $[8,9]$. This led to the FDA extending the EUA for Pfizer-BioNTech and Moderna mRNA vaccines to include additional doses in immunocompromised patients. However, these results may not be generalizable to CLL, and additional studies are needed to better define vaccine responses in the CLL patient population, including the role of mixing mRNA vaccination with other vaccine formulations, such as the adenovirus vectored vaccine Ad26COV2.s, commonly known as the Johnson and Johnson (J\&J) vaccine.

\section{Case Report}

Here we describe two CLL patients who "self-referred" to outside pharmacies for an additional vaccination with the J\&J COVID-19 vaccine following 2 doses of the BNT162b2 vaccine (Pfizer-BioNTech). Both patients had previously been enrolled as study subjects in an IRB-approved observational study (OHSU IRB\# 21230) to investigate immune response following COVID-19 vaccination. The additional J\&J dose was subsequently self-reported to the study team. On initial enrollment, demographics, CLL disease characteristics, and treatment details were collected (Table 1), and baseline laboratory values were obtained, including semiquantitative SARS-CoV-2 spike antibody titer, serum IgG, a complete blood count, and multicolor flow cytometry measuring immune cell populations (Table 1). Whole blood was collected for additional serologic and cellular studies.

SARS-CoV-2 spike receptor binding domain (RBD)specific antibody levels were tested by ELISA and endpoint titers were calculated as previously described [10]. In addition, baseline PBMC samples were functionally tested for the presence of SARS-CoV-2 spike RBD-specific memory B-cells (MBCs) by limiting dilution assay as previously described $[11,12]$. Briefly, PBMCs were serially diluted and incubated with a stimulation cocktail in which the MBCs present within PBMCs differentiated and became antibodysecreting cells. The supernatants were collected 7 days later and assayed for antigen specificity by RBD-ELISA. This allows one to functionally test MBC-derived antibodies and back-calculate the frequency of total IgG-secreting MBCs as well as RBD-specific MBCs $[11,12]$. In addition, CD4+ and $\mathrm{CD} 8+\mathrm{T}$-cells were also functionally assessed for the presence of IFN' $\gamma$ and TNF $\alpha$ secretion following spike proteinderived peptide stimulation. Briefly, PBMCs were stimulated with 2 peptide pools of overlapping (10AA) 17mers representing the SARS-CoV-2 spike protein (BEI Resources). Following stimulation, the cells were stained as previously described $[13,14]$. Data was acquired on an LSR Fortessa (Becton Dickenson) and analyzed using FlowJo software. Cytokine expression in medium-alone cultures was subtracted from peptide-stimulated cultures to calculate peptide-specific cytokine expression. Responses to both peptide pools were added together to yield the total frequency of SARS-CoV-2-specific cytokine-producing CD4+ and CD8+ T cells.
Neither subject had prevaccination B-cell responses as measured by RBD-specific antibodies or MBCs. Neither had a virus-specific CD8+ response at baseline. While Subject 2 had spike peptide-reactive CD4+ T-cells at baseline, these cells were unresponsive and did not expand following vaccination. In contrast, CD8+ responses were observed after mRNA vaccination in both subjects (Figure 1). It has previously been reported that SARS-CoV-2 naive individuals may have preexisting cross-reactivity to SARS-CoV-2 peptides through prior infection by common cold coronaviruses: SARS-CoV-2 specific CD4+ T-cells have been identified in $20-50 \%$ of people without SARS-CoV-2 exposure or vaccination [15].

Approximately four weeks after initial vaccination, neither subject had detectable RBD-specific SARS-CoV-2 antibodies or MBCs. Both had measurable vaccine-induced CD8+ T-cell responses following mRNA vaccination, although CD4+ responses did not appear to increase above baseline (Figure 1).

Subject 1 received the J\&J vaccine 104 days and Subject 2 received 81 days after completion of the BNT162b2 vaccine series. Following J\&J vaccination, additional samples were obtained from Subject 1, 30 days after the third vaccine, and Subject 2, 27 days following the third vaccine. Interestingly, Subject 1 had undetectable RBD-specific antibodies, RBDspecific MBCs, and virus-specific CD4+ T-cells after the initial vaccination series. However, following an additional vaccination, all three measures increased above the limit of detection: RBD-ELISA titer of 625, RBD-specific MBC frequency of $3.6 / 10^{6} \mathrm{~B}$-cells, and 166 spike-specific CD4+ $\mathrm{T}$-cells $/ 10^{6}$, and a spike-specific CD8+ T-cell response that remained stable and did not boost appreciably following the $3^{\text {rd }}$ vaccination (Figure 1 ). Subject 2 did not seroconvert or have detectable virus-specific MBCs after their primary mRNA vaccination series; however, they had a spike-specific CD8+ T-cell response that was further boosted after a $3^{\text {rd }}$ dose and a virus-specific CD4+ response that did not change following the original vaccine series or the $3^{\text {rd }}$ dose of J\&J.

\section{Discussion}

Other than subject age (60s vs 80 s), the most notable difference between the subjects' baseline characteristics (Table 1) is that Subject 1 was treatment naive, while Subject 2 had undergone previous treatment (6 years ago) with obinutuzumab, an anti$\mathrm{CD} 20 \mathrm{mAb}$, and is currently on active treatment with ibrutinib since 2017. Both had baseline B-cell frequencies outside of the normal range, with Subject 1 exhibiting a low percentage of naïve B-cells (0.092) and a high percentage of MBCs (59.1), while Subject 2 had a low percentage of naïve B-cells (11.37) and MBCs (0.45). Although Subject 2 had mild hypogammaglobulinemia, neither had a history of recurring infections or a need for IgG supplementation. Levels of baseline CD4+ and CD8+ T-cells (absolute values) were also normal in each subject prior to vaccination (data not shown). Both had very low percentages of naïve $B$ cells which could explain the initial poor response to vaccination. The significance of the increased percentage of MBCs in Subject 1 is unclear, but does suggest some broader preservation of normal B cell maturation and 


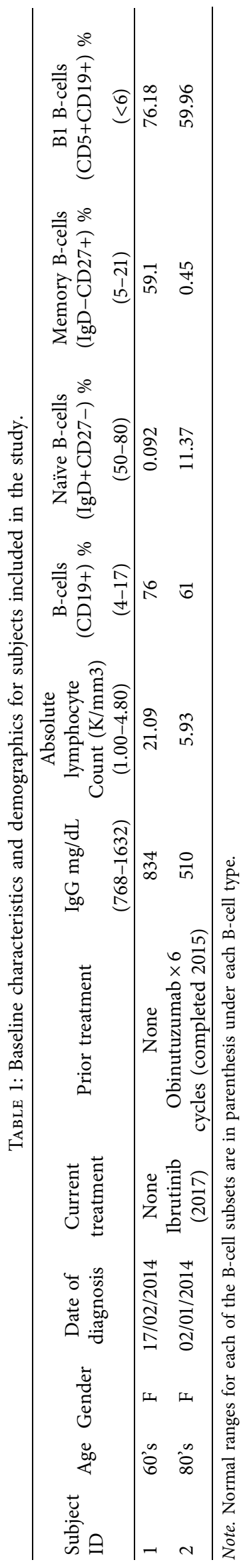



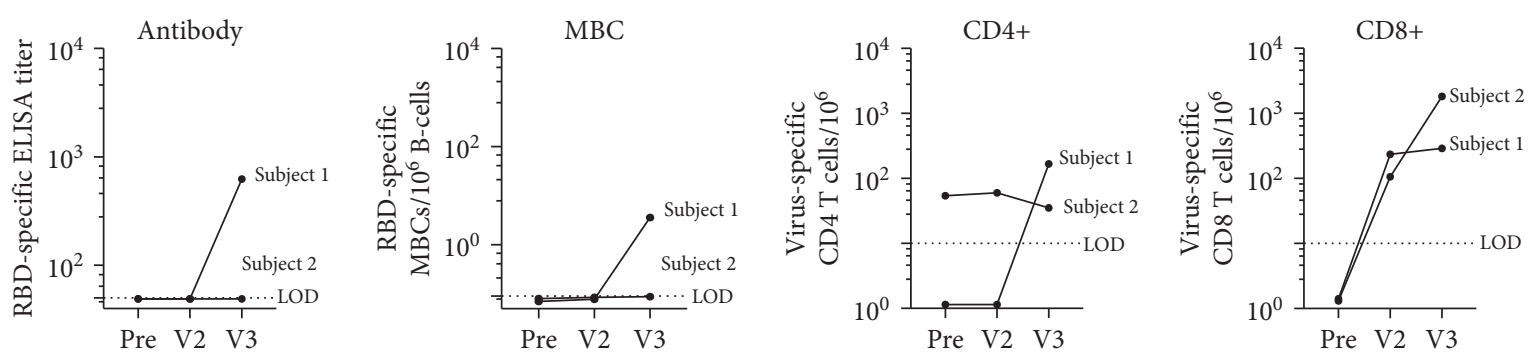

FIGURE 1: Immune response to COVID-19 vaccination in CLL subjects. RBD-specific antibody titer. Subjects without a detectable antibody titer ( $<1: 50$ serum dilution) were assigned a value of 49 . The limit of detection (LOD) is 50 . Frequency of RBD-specific MBCs per $10^{6}$ CD19+ B-cells following ex vivo stimulation. Subjects who did not have a detectable response were assigned a value between 0.07 and 0.09 . The limit of detection (LOD) is 0.1. SARS-CoV-2 spike peptide-reactive CD4 and CD8 T-cells are defined as double positive for IFN $\gamma$ and TNF $\alpha$ cytokine secretion. Patients who did not have a detectable T-cell response were assigned an arbitrary number less than 2 . The limit of detection (LOD) is 10. Visit 1 (pre) blood draw was taken 21 and 40 days prior to the Pfizer vaccine series (2-doses). Visit 2 (V2) blood draw was taken 33 and 24 days post vaccination, and Visit 3 (V3) was drawn 30 and 27 days after the 3rd vaccination with J\&J.

immune function. Although Subject 1 did have an immune response, antibody levels were relatively low as compared to some of the levels observed in immunocompetent postvaccine populations [16] and certain CLL populations [4]. The clinical significance of specific antibody levels remains unknown.

Active treatment with Bruton's tyrosine kinase (BTK) inhibitors like ibrutinib may have a profound impact on B-cell survival, differentiation, and production of antibodies as the absence of intact BTK-dependent B-cell receptor mediated signaling prevents B-cells from differentiating into mature peripheral B-cells. Immune responses following vaccination or natural infection are limited in these patients [17]. Recall to antigens encountered prior to treatment appears to remain largely intact; however, response to novel antigens encountered during treatment seems to be abrogated. Subject 2 has been on ibrutinib for over four years. The impact of prolonged treatment vs. shorter-term BTK inhibition on immune responses is unknown. However, clinical data [18] suggest some improvement in humoral immunity with prolonged ( $>6$ months) treatment. T-cells are also disrupted in individuals with CLL and even further disrupted with BTK treatment [19]. In the cases presented here, both subjects did have an increase in virus-specific CD8+ T-cells; however, the significance is unclear in terms of protection, as neutralizing antibodies are often viewed as the correlate of protection against COVID-19.

\section{Conclusion}

The results of this study, however small, provide initial evidence that a $3^{\text {rd }}$ vaccination against COVID-19 with the heterotypic vaccine Ad26COV2.s results in an immune response that was not observed following the recommended 2-dose mRNA vaccination series. This is especially promising news to subjects who are treatment naïve, not currently in active treatment, or who may consider vaccination before beginning active treatment.

\section{Data Availability}

The data used to support the findings of this study are included within the article.

\section{Disclosure}

The funders had no involvement in study design; in the collection, analysis, and interpretation of data; in the writing of the report; or in the decision to submit the article for publication.

\section{Conflicts of Interest}

Stephen E. Spurgeon is consultant at Velos Bio, Karyopharm, Genentech, Janssen, and Pharmacyclics and researcher at Acerta Pharma, Astrazeneca, Beigene, Bristol Myers Squibb, Genentech, Gilead Sciences, Ionis, and Janssen. All other authors have no conflicts of interest.

\section{Authors' Contributions}

Zoe L. Lyski contributed to formal analysis, investigation, and visualization, prepared the original draft, and edited and reviewed the manuscript. Myung Sun Kim was responsible for investigation, project administration, visualization, and resources. David Xthona Lee contributed to investigation and formal analysis. David Sampson was responsible for project administration and resources. Hans P. Raué contributed to formal analysis and investigation and edited and reviewed the manuscript. Vikram Raghunathan was responsible for investigation, project administration, and recourses. Debbie Ryan was responsible for project administration and resources. Amanda E. Brunton performed formal analysis and was responsible for resources. Mark K. Slifka was involved in funding acquisition and supervision and reviewed and edited the manuscript. William B. Messer was responsible for conceptualization, funding acquisition and supervision and reviewed and edited the manuscript. Stephen E. Spurgeon was responsible for conceptualization, funding acquisition, project administration, and supervision, prepared the original draft and reviewed and edited the manuscript.

\section{Acknowledgments}

The authors would like to thank the subjects for participating in this research study. This work was funded in part by the 
National Institute of Allergy and Infectious Diseases NIAID 1R01AI145835 (WBM), US National Institute of Health grant P51 OD011092 (MKS), and endowed funds from the Knight Cancer Institute's Scholar Award for Leukemia and Lymphoma Research (SES).

\section{References}

[1] P. Wadhwa and V. Morrison, "Infectious complications of chronic lymphocytic leukemia," Seminars in Oncology, vol. 33, no. 2, pp. 240-249, 2006.

[2] L. Scarfò, T. Chatzikonstantinou, G. M. Rigolin et al., "COVID-19 severity and mortality in patients with chronic lymphocytic leukemia: a joint study by ERIC, the European Research Initiative on CLL, and CLL Campus," Leukemia, vol. 34, no. 9, pp. 2354-2363, 2020.

[3] J. A. Whitaker, S. A. Parikh, T. D. Shanafelt et al., "The humoral immune response to high-dose influenza vaccine in persons with monoclonal B-cell lymphocytosis (MBL) and chronic lymphocytic leukemia (CLL)," Vaccine, vol. 39, no. 7, pp. 1122-1130, 2021.

[4] F. P. Polack, S. J. Thomas, N. Kitchin et al., "Safety and efficacy of the BNT162b2 mRNA covid-19 vaccine," New England Journal of Medicine, vol. 383, no. 27, pp. 2603-2615, 2020.

[5] Y. Herishanu, I. Avivi, A. Aharon et al., "Efficacy of the BNT162b2 mRNA COVID-19 vaccine in patients with chronic lymphocytic leukemia," Blood, vol. 137, no. 23, pp. 3165-3173, 2021.

[6] L. E. Roeker, D. A. Knorr, M. C. Thompson et al., "COVID-19 vaccine efficacy in patients with chronic lymphocytic leukemia," Leukemia, vol. 35, no. 9, pp. 2703-2705, 2021.

[7] Z. Lyski, M. S. Kim, D. Xthona Lee et al., "Cellular and humoral Immune response to mRNA COVID-19 vaccination in subjects with chronic lymphocytic leukemia," medRxiv, 2021.

[8] N. Kamar, F. Abravanel, O. Marion, C. Couat, J. Izopet, and A. Del Bello, "Three doses of an mRNA covid-19 vaccine in solid-organ transplant recipients," New England Journal of Medicine, vol. 385, no. 7, pp. 661-662, 2021.

[9] R. T. Shroff, P. Chalasani, R. Wei et al., "Immune responses to two and three doses of the BNT162b2 mRNA vaccine in adults with solid tumors," Nature Medicine, vol. 27, no. 11, pp. 2002-2011, 2021

[10] A. Thomas, W. B. Messer, D. E. Hansel et al., "Establishment of monoclonal antibody standards for quantitative serological diagnosis of SARS-CoV-2 in low-incidence settings," Open Forum Infectious Diseases, vol. 8, no. 3, p. ofab061, 2021.

[11] Z. L. Lyski, A. E. Brunton, M. I. Strnad et al., "SARS-CoV-2 specific memory B-cells from individuals with diverse disease severities recognize SARS-CoV-2 variants of concern," The Journal of Infectious Diseases, 2021.

[12] I. J. Amanna and M. K. Slifka, "Quantitation of rare memory B cell populations by two independent and complementary approaches," Journal of Immunological Methods, vol. 317, no. 1-2, pp. 175-185, 2006.

[13] H. P. Raué and M. K. Slifka, "Pivotal advance: CTLA-4+ T cells exhibit normal antiviral functions during acute viral infection," Journal of Leukocyte Biology, vol. 81, no. 5, pp. 11651175, 2007.

[14] H. P. Raué and M. K. Slifka, "CD8+ T cell immunodominance shifts during the early stages of acute LCMV infection independently from functional avidity maturation," Virology, vol. 390, no. 2, pp. 197-204, 2009.
[15] J. Mateus, A. Grifoni, A. Tarke et al., "Selective and crossreactive SARS-CoV-2 T cell epitopes in unexposed humans," Science, vol. 370, no. 6512, pp. 89-94, 2020.

[16] T. A. Bates, H. C. Leier, Z. L. Lyski et al., "Age-dependent neutralization of SARS-CoV-2 and P.1 variant by vaccine immune serum samples," JAMA, vol. 326, 2021.

[17] C. Pleyer, M. A. Ali, J. I. Cohen et al., "Effect of Bruton tyrosine kinase inhibitor on efficacy of adjuvanted recombinant hepatitis B and zoster vaccines," Blood, vol. 137, no. 2, pp. 185-189, 2021.

[18] C. Sun, X. Tian, Y. S. Lee et al., "Partial reconstitution of humoral immunity and fewer infections in patients with chronic lymphocytic leukemia treated with ibrutinib," Blood, vol. 126, no. 19, pp. 2213-2219, 2015.

[19] P. M. Roessner and M. Seiffert, "T-cells in chronic lymphocytic leukemia: guardians or drivers of disease?" Leukemia, vol. 34 , no. 8 , pp. $2012-2024,2020$. 